\title{
Dynamic stabilization of foam films with acoustic sound
}

Chun Yong Ng, Hangil Park, Liguang Wang*

2 pages, 0 figure, 1 table 
Table S1. Corresponding to Fig. 4, the lifetime of foam films formed from $1 \times 10^{-4} \mathrm{M}$ SDS with $0.1 \mathrm{M} \mathrm{NaCl}$ at a range of sound frequencies $\left(f_{\mathrm{s}}\right)$ and amplitudes $\left(A_{\mathrm{s}}\right)$. The film radius was $400 \pm 40 \mu \mathrm{m}$. The three levels of film stability are highlighted in green (unstable film), red (meta-stable film) and blue (stable film). The lifetime of the film at static condition was $19 \mathrm{~s}$. The errors following the lifetime represents a standard error obtained from three independent tests.

\begin{tabular}{|c|c|c|c|c|c|c|c|c|}
\hline$f_{\mathrm{s}}(\mathrm{kHz})$ & $A_{\mathrm{s}}(\mathrm{dB})$ & Lifetime (s) & $f_{\mathrm{s}}(\mathrm{kHz})$ & $A_{\mathrm{s}}(\mathrm{dB})$ & Lifetime (s) & $f_{\mathrm{s}}(\mathrm{kHz})$ & $A_{\mathrm{s}}(\mathrm{dB})$ & Lifetime (s) \\
\hline 1.0 & 76 & $19 \pm 1$ & 8.8 & 75 & $21 \pm 2$ & 12.0 & 73 & $21 \pm 1$ \\
\hline 1.0 & 92 & $19 \pm 1$ & 8.8 & 87 & $22 \pm 1$ & 12.0 & 80 & $18 \pm 1$ \\
\hline 1.0 & 104 & $18 \pm 2$ & 8.8 & 100 & $18 \pm 2$ & 12.0 & 100 & $14 \pm 1$ \\
\hline 1.0 & 114 & $17 \pm 1$ & 8.8 & 109 & $17 \pm 1$ & 12.0 & 102 & $13 \pm 1$ \\
\hline 1.0 & 117 & $16 \pm 2$ & 8.8 & 112 & $16 \pm 1$ & 12.0 & 105 & $15 \pm 3$ \\
\hline 1.0 & 123 & $16 \pm 4$ & 8.8 & 116 & $37 \pm 3$ & 12.0 & 109 & $22 \pm 2$ \\
\hline 2.0 & 75 & $19 \pm 1$ & 8.9 & 75 & $19 \pm 1$ & 13.0 & 73 & $20 \pm 1$ \\
\hline 2.0 & 93 & $18 \pm 1$ & 8.9 & 87 & $20 \pm 1$ & 13.0 & 81 & $20 \pm 1$ \\
\hline 2.0 & 105 & $18 \pm 2$ & 8.9 & 100 & $17 \pm 1$ & 13.0 & 94 & $14 \pm 5$ \\
\hline 2.0 & 115 & $15 \pm 1$ & 8.9 & 109 & $17 \pm 1$ & 13.0 & 104 & $14 \pm 3$ \\
\hline 2.0 & 118 & $17 \pm 2$ & 8.9 & 112 & $33 \pm 4$ & 13.0 & 108 & $18 \pm 1$ \\
\hline 2.0 & 124 & $13 \pm 1$ & 8.9 & 116 & $62 \pm 10$ & 13.0 & 111 & $21 \pm 1$ \\
\hline 3.0 & 79 & $18 \pm 1$ & 9.0 & 74 & $19 \pm 1$ & 14.0 & 73 & $17 \pm 1$ \\
\hline 3.0 & 97 & $21 \pm 1$ & 9.0 & 87 & $18 \pm 1$ & 14.0 & 84 & $18 \pm 1$ \\
\hline 3.0 & 109 & $17 \pm 2$ & 9.0 & 96 & $14 \pm 2$ & 14.0 & 93 & $16 \pm 3$ \\
\hline 3.0 & 119 & $18 \pm 4$ & 9.0 & 109 & $18 \pm 1$ & 14.0 & 102 & $15 \pm 1$ \\
\hline 3.0 & 122 & $13 \pm 2$ & 9.0 & 112 & $28 \pm 4$ & 14.0 & 107 & $16 \pm 1$ \\
\hline 3.0 & 128 & $13 \pm 1$ & 9.0 & 115 & $77 \pm 7$ & 14.0 & 114 & 300 \\
\hline 4.0 & 76 & $20 \pm 1$ & 9.1 & 75 & $20 \pm 1$ & 15.0 & 76 & $20 \pm 1$ \\
\hline 4.0 & 95 & $19 \pm 1$ & 9.1 & 87 & $21 \pm 1$ & 15.0 & 82 & $18 \pm 1$ \\
\hline 4.0 & 106 & $13 \pm 2$ & 9.1 & 99 & $18 \pm 2$ & 15.0 & 96 & $14 \pm 4$ \\
\hline 4.0 & 116 & $15 \pm 1$ & 9.1 & 108 & $17 \pm 1$ & 15.0 & 105 & $18 \pm 2$ \\
\hline 4.0 & 120 & $15 \pm 1$ & 9.1 & 111 & $31 \pm 3$ & 15.0 & 111 & $22 \pm 2$ \\
\hline 4.0 & 125 & $13 \pm 1$ & 9.1 & 115 & $53 \pm 12$ & 15.0 & 115 & 300 \\
\hline 5.0 & 74 & $18 \pm 2$ & 9.3 & 74 & $19 \pm 2$ & 16.0 & 77 & $18 \pm 2$ \\
\hline 5.0 & 93 & $19 \pm 1$ & 9.3 & 87 & $20 \pm 1$ & 16.0 & 85 & $18 \pm 1$ \\
\hline 5.0 & 104 & $16 \pm 1$ & 9.3 & 99 & $18 \pm 1$ & 16.0 & 98 & $20 \pm 5$ \\
\hline 5.0 & 113 & $14 \pm 2$ & 9.3 & 107 & $18 \pm 1$ & 16.0 & 107 & $18 \pm 1$ \\
\hline 5.0 & 117 & $13 \pm 2$ & 9.3 & 111 & $33 \pm 7$ & 16.0 & 113 & 300 \\
\hline 5.0 & 122 & $10 \pm 1$ & 9.3 & 115 & $39 \pm 7$ & 16.0 & 117 & 300 \\
\hline 6.0 & 75 & $20 \pm 1$ & 9.5 & 74 & $19 \pm 1$ & 17.0 & 78 & $21 \pm 2$ \\
\hline 6.0 & 89 & $19 \pm 2$ & 9.5 & 86 & $20 \pm 1$ & 17.0 & 85 & $20 \pm 1$ \\
\hline 6.0 & 100 & $12 \pm 2$ & 9.5 & 99 & $17 \pm 1$ & 17.0 & 99 & $22 \pm 4$ \\
\hline 6.0 & 109 & $13 \pm 2$ & 9.5 & 107 & $18 \pm 1$ & 17.0 & 107 & 300 \\
\hline 6.0 & 113 & $10 \pm 2$ & 9.5 & 111 & $18 \pm 2$ & 17.0 & 113 & 300 \\
\hline 6.0 & 116 & $10 \pm 1$ & 9.5 & 113 & $11 \pm 1$ & 17.0 & 117 & 300 \\
\hline 7.0 & 74 & $18 \pm 1$ & 10.0 & 74 & $20 \pm 1$ & 18.0 & 75 & $22 \pm 1$ \\
\hline 7.0 & 85 & $20 \pm 1$ & 10.0 & 88 & $20 \pm 1$ & 18.0 & 84 & $21 \pm 1$ \\
\hline 7.0 & 95 & $17 \pm 1$ & 10.0 & 96 & $13 \pm 2$ & 18.0 & 100 & $18 \pm 2$ \\
\hline 7.0 & 103 & $16 \pm 3$ & 10.0 & 106 & $11 \pm 1$ & 18.0 & 107 & 300 \\
\hline 7.0 & 108 & $13 \pm 1$ & 10.0 & 111 & $14 \pm 1$ & 18.0 & 113 & 300 \\
\hline 7.0 & 112 & $12 \pm 2$ & 10.0 & 113 & $12 \pm 3$ & 18.0 & 116 & 300 \\
\hline 8.0 & 75 & $18 \pm 2$ & 11.0 & 74 & $20 \pm 1$ & 19.0 & 73 & $20 \pm 3$ \\
\hline 8.0 & 87 & $17 \pm 1$ & 11.0 & 87 & $20 \pm 1$ & 19.0 & 84 & $18 \pm 1$ \\
\hline 8.0 & 99 & $13 \pm 1$ & 11.0 & 101 & $17 \pm 2$ & 19.0 & 100 & $15 \pm 4$ \\
\hline 8.0 & 109 & $15 \pm 4$ & 11.0 & 104 & $16 \pm 1$ & 19.0 & 107 & $26 \pm 2$ \\
\hline 8.0 & 113 & $13 \pm 3$ & 11.0 & 105 & $18 \pm 1$ & 19.0 & 113 & 300 \\
\hline 8.0 & 118 & $13 \pm 1$ & 11.0 & 107 & $21 \pm 3$ & 19.0 & 115 & 300 \\
\hline 8.7 & 75 & $20 \pm 1$ & & & & & & \\
\hline 8.7 & 88 & $18 \pm 1$ & & & & & & \\
\hline 8.7 & 101 & $17 \pm 2$ & & & & & & \\
\hline 8.7 & 109 & $18 \pm 2$ & & & & & & \\
\hline 8.7 & 113 & $16 \pm 1$ & & & & & & \\
\hline 8.7 & 117 & $13 \pm 2$ & & & & & & \\
\hline
\end{tabular}

